\title{
Effects of Preoperative Malnutrition on Postoperative Surgical Outcomes and Quality of Life of Elderly Patients with Periampullary Neoplasms: A Single-Center Prospective Cohort Study
}

\author{
Eunjung Kim ${ }^{1}$, Doo-Ho Lee ${ }^{2}$, and Jin-Young Jang ${ }^{2}$ \\ ${ }^{1}$ Department of Nursing, Graduate School, Kyung Hee University, and ${ }^{2}$ Department of Surgery and Cancer Research Institute, Seoul National \\ University College of Medicine, Seoul, Korea
}

Background/Aims: We investigated the effect of preoperative malnutrition on postoperative surgical outcomes in elderly patients undergoing pancreatoduodenectomy for periampullary neoplasms. Methods: This prospective cohort study enrolled 154 patients aged $\geq 65$ years with periampullary neoplasms. Using the Mini Nutritional Assessment tool, patients were categorized into three groups according to their preoperative nutritional status: well-nourished (13.0\%), at-risk-of-malnutrition (59.7\%), and malnourished (27.3\%). Results: Significant intergroup differences were observed in preoperative body mass index $\left(25.6 \pm 2.4 \mathrm{~kg} / \mathrm{m}^{2}\right.$ [wellnourished] vs $23.4 \pm 2.6 \mathrm{~kg} / \mathrm{m}^{2}$ [at-risk-of-malnutrition] vs $21.1 \pm 2.8 \mathrm{~kg} / \mathrm{m}^{2}$ [malnourished], $\mathrm{p}<0.001$ ). The overall morbidity significantly differed between the well-nourished and malnourished groups ( $20 \%$ vs $50.0 \%, p=0.024$ ). The rates of clinically significant postoperative pancreatic fistula were significantly different among groups $(p=0.035)$. Univariate and multivariate analyses showed that the at-risk-ofmalnutrition or malnourished status (hazard ratio [HR], 3.45; $p=0.037$ ) and intraoperative blood loss (HR, 1.01; $p=0.040)$ significantly affected the overall postoperative morbidity in elderly patients. Conclusions: Before surgery, $87.0 \%$ of patients were classified into the at-risk-of-malnutrition or malnourished group. Compared with well-nourished patients, patients with nutritional issues showed a higher overall surgical morbidity. Improved preoperative nutritional status leads to favorable surgical outcomes in elderly patients. (Gut Liver 2019;13:690-697)

Key Words: Pancreatoduodenectomy; Nutritional status; Elderly

\section{INTRODUCTION}

Periampullary neoplasms comprise heterogeneous lesions including benign and malignant neoplasms of the pancreatic head, ampulla of Vater (AoV), distal common bile duct (CBD), and duodenum. Regardless of the tumor origin, pancreatoduodenectomy (PD) is the standard treatment for periampullary neoplasms. ${ }^{1}$ Surgical mortality rates have declined following advances in surgical techniques and the perioperative care associated with PD. However, the complexity of the surgical procedure leads to considerable morbidity after PD. ${ }^{2,3}$ With the increase in life expectancy in the fast-growing population of elderly patients, pancreatic procedures are increasingly being performed in this patient population. However, functional decline and comorbidities increase the risk of postoperative complications after major pancreatic resection in elderly patients. ${ }^{4}$

Tumor growth in patients with periampullary neoplasms including pancreatic cancer leads to obstruction of the pancreatic duct and/or CBD. ${ }^{1,2}$ As the reduction of digestive juice hinders nutrient absorption, most patients present to the hospital with malnutrition associated with weight loss. Patients with periampullary neoplasm should undergo Whipple's operation, which entails resection of the pancreatic head, duodenum, CBD, and/or pylorus of the stomach; these organs play a major role in digestion. However, few prospective studies have reported the use of nutritional index before and after Whipple's operation. Hence, it is important to classify elderly patients with nutritional risk who are scheduled for surgery to treat periampullary neoplasm according to their nutritional status, and to provide them with nutritional education and intervention to improve their preop-

\footnotetext{
Correspondence to: Jin-Young Jang

Department of Surgery and Cancer Research Institute, Seoul National University Hospital, Seoul National University College of Medicine, 101 Daehak-ro, Jongno-gu, Seoul 03080, Korea

Tel: +82-2-2072-2194, Fax: +82-2-741-2194, E-mail: jangjy4@gmail.com

Received on October 17, 2018. Revised on November 30, 2018. Accepted on December 18, 2018. Published online May 27, 2019.

pISSN 1976-2283 eISSN 2005-1212 https://doi.org/10.5009/gnl18469

Eunjung Kim and Doo-Ho Lee contributed equally to this work as first authors.

*Current affiliation of Doo-Ho Lee is Department of Surgery, Gil Medical Center, Gachon University, Incheon, Korea.

(a) This is an Open Access article distributed under the terms of the Creative Commons Attribution Non-Commercial License (http://creativecommons.org/licenses/by-nc/4.0) which permits unrestricted non-commercial use, distribution, and reproduction in any medium, provided the original work is properly cited
} 
erative nutritional status.

Quality of life (QOL) is a reference tool to assess the quality of care, and it serves as an important appraisal instrument in geriatric assessment. ${ }^{5}$ Impaired QOL is associated with malnutrition. $^{6-8}$

This study aimed to categorize all elderly patients according to their preoperative nutritional status, using the Mini Nutritional Assessment (MNA) tool to evaluate the effect of preoperative nutritional status on postoperative morbidity, cognitive function, and the QOL of elderly patients with periampullary neoplasms. We used a prospective database from the Seoul National University Hospital (SNUH), Seoul, Korea.

\section{MATERIALS AND METHODS}

\section{Study design}

We performed a prospective cohort study using prospectively recorded information of patients registered in a clinical database at SNUH between January 2008 and December 2014. The study was approved by the Institutional Review Board of SNUH (IRB No. 0801-030-232). Informed consent was obtained from all patients before enrollment. This study was performed according to the ethical standards of the Helsinki Declaration.

\section{Patient classification, and inclusion and exclusion criteria}

Patients aged $\geq 65$ years at the time of diagnosis who underwent pylorus-preserving pancreatoduodenectomy (PPPD) or PD to treat periampullary neoplasms, those without any severe comorbidities such as liver cirrhosis or end-stage renal disease, and those who provided written informed consent for participation were included in the study. Patients aged $<65$ years, those undergoing palliative resection, those showing metastasis or recurrence, those receiving neoadjuvant treatment, those with a history of other abdominal operations (including gastrectomy or colectomy), those with cerebrovascular accidents (these could affect cognitive function), those with an American Society of Anesthesiologists (ASA) classification score >III, and those in whom information regarding MNA was unavailable were excluded. Finally, we categorized 154 patients into three groups based on their preoperative nutritional status using MNA as: well- nourished, at-risk-of-malnutrition, and malnourished (Fig. 1).

\section{Primary and secondary outcomes of the study}

This study was designed to evaluate postoperative overall morbidity according to the nutritional status of patients who underwent PD or PPPD for periampullary neoplasms. Secondary outcomes included complications of Clavien-Dindo criteria grade IIIa or worse, rate of perioperative pancreatic fistula (POPF), cognitive score, QOL score, and length of hospital stay. In addition, univariate and multivariate logistic regression analyses were performed to evaluate the risk factors affecting overall postoperative morbidity.

\section{Data extraction}

Data were collected before and after surgery. All patients were enrolled within 24 hours of admission for an elective operation. Nutritional status was classified using the MNA, which comprised 16 questionnaires under four sections: $:^{9,10}$ anthropometric, general, dietary, and subjective assessment. All answers were expressed as numerical values, which were converted into scores, with a maximum of 28 points. Patients completed selfreported questionnaires. The nutritional status was assigned a score, with patients scoring $>22$ considered well-nourished, those scoring 15 to 21.5 considered at-risk-of-malnutrition, and those scoring $<15$ considered malnourished. ${ }^{9}$

Data regarding the cognitive function and QOL of the patients were obtained before surgery, using questionnaires. Cognitive function was assessed using a 4-point scale cognitive function questionnaire based on the European Organization for Research and Treatment of Cancer (EORTC QLQ-C30). ${ }^{11}$ QOL was assessed using the global health status/QOL questionnaires from the EORTC QLQ-C30. Overall, the self-reported QOL questionnaires comprised responses categorized on a 7-point scale. Transformation of raw scores into a 0 to 100 scale and adjustments of missing data were performed according to the EORTC QLQ-C30 scoring manual.

Patient demographics, tumor location, type of operation, length of hospitalization, pathological report, and postoperative morbidity were recorded for each patient. Postoperative morbidity was graded using the Clavien-Dindo classification. ${ }^{12}$

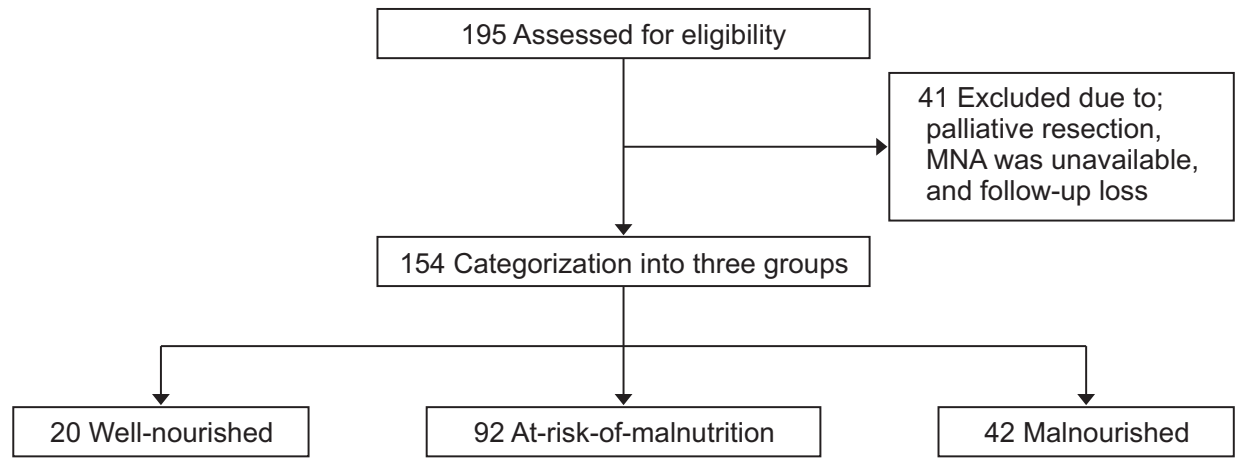

Fig. 1. Diagram for patient disposition. MNA, Mini Nutritional Assessment. 
POPF was defined according to the International Study Group of Pancreatic Fistula criteria, ${ }^{13}$ which define a pancreatic fistula as a measurable volume of drainage fluid with an amylase concentration $>3$ times the upper limit of normal after postoperative day 3. Three grades of POPF are defined, on the basis of the clinical effect of POPF on the postoperative course of the patient. Delayed gastric emptying was defined as nasogastric drainage for $>10$ days, the need to reinsert a nasogastric tube because of vomiting, or the inability to tolerate a semisolid diet 14 days after surgery. ${ }^{14}$

Table 1. Patient Demographics and Pathologic Outcomes According to Preoperative Nutritional Status

\begin{tabular}{|c|c|c|c|c|c|c|c|c|}
\hline \multirow{2}{*}{ Parameter } & \multirow{2}{*}{$\begin{array}{l}\text { Overall } \\
(n=154)\end{array}$} & \multirow{2}{*}{$\begin{array}{c}\text { Group A } \\
(n=20)\end{array}$} & \multirow{2}{*}{$\begin{array}{c}\text { Group B } \\
(n=92)\end{array}$} & \multirow{2}{*}{$\begin{array}{l}\text { Group C } \\
(n=42)\end{array}$} & \multicolumn{4}{|c|}{$\mathrm{p}$-value } \\
\hline & & & & & Total & A vs B & A vs C & B vs C \\
\hline Age, yr & $71.0 \pm 4.5$ & $69.4 \pm 3.5$ & $71.2 \pm 4.5$ & $71.5 \pm 4.9$ & 0.235 & 0.228 & 0.179 & 0.909 \\
\hline Sex & & & & & 0.185 & 0.124 & 0.068 & 0.530 \\
\hline Male & $80(51.9)$ & $14(70.0)$ & $47(51.1)$ & $19(45.2)$ & & & & \\
\hline Preoperative diabetes mellitus & $46(29.9)$ & $5(25.0)$ & $29(31.5)$ & $12(28.6)$ & 0.828 & 0.565 & 0.768 & 0.731 \\
\hline Preoperative BMI, kg/m² & $23.0 \pm 2.9$ & $25.6 \pm 2.4$ & $23.4 \pm 2.6$ & $21.1 \pm 2.8$ & $<0.001^{*}$ & $0.003^{*}$ & $<0.001^{*}$ & $<0.001^{*}$ \\
\hline Transferrin, mg/dL & $217.8 \pm 47.8$ & $223.5 \pm 31.8$ & $223.3 \pm 49.8$ & $203.9 \pm 47.4$ & 0.103 & 0.998 & 0.212 & 0.123 \\
\hline Prealbumin, mg/dL & $22.6 \pm 7.2$ & $23.6 \pm 6.1$ & $22.1 \pm 6.0$ & $23.1 \pm 9.6$ & 0.718 & 0.803 & 0.995 & 0.939 \\
\hline Albumin, g/dL & $3.7 \pm 0.4$ & $3.9 \pm 0.3$ & $3.7 \pm 0.4$ & $3.6 \pm 0.4$ & 0.125 & 0.280 & 0.071 & 0.618 \\
\hline Cognitive score & $86.0 \pm 17.1$ & $91.7 \pm 10.3$ & $86.6 \pm 17.4$ & $82.1 \pm 18.1$ & 0.127 & 0.281 & 0.078 & 0.456 \\
\hline QOL score & $52.5 \pm 27.8$ & $71.5 \pm 18.5$ & $57.9 \pm 25.9$ & $31.1 \pm 23.2$ & $<0.001^{*}$ & $0.032^{*}$ & $<0.001^{*}$ & $<0.001^{*}$ \\
\hline Pancreas texture & & & & & 0.164 & 0.958 & 0.205 & 0.062 \\
\hline Soft & $112(72.7)$ & $15(75.0)$ & 68 (73.9) & $29(69.0)$ & & & & \\
\hline Hard & $42(27.3)$ & $5(25.0)$ & $24(26.1)$ & $13(31.0)$ & & & & \\
\hline Intraoperative blood loss, $\mathrm{mL}$ & $350 \pm 266$ & $326 \pm 150$ & $349 \pm 305$ & $364 \pm 214$ & 0.519 & 0.629 & 0.422 & 0.733 \\
\hline Operation time, $\min$ & $305 \pm 90$ & $337 \pm 79$ & $302 \pm 97$ & $298 \pm 76$ & 0.244 & 0.097 & 0.081 & 0.832 \\
\hline Main pancreatic duct diameter, mm & & & & & 0.164 & 0.920 & 0.632 & 0.560 \\
\hline$\geq 3$ & $91(59.1)$ & $11(55.0)$ & $50(54.3)$ & $30(71.4)$ & & & & \\
\hline$<3$ & $63(40.9)$ & $9(45.0)$ & $42(45.7)$ & $12(28.6)$ & & & & \\
\hline Operation & & & & & 0.555 & 0.309 & 0.280 & 0.845 \\
\hline Pylorus preserving & $135(87.7)$ & $19(95.0)$ & $80(87.0)$ & 36 (85.7) & & & & \\
\hline pancreatoduodenectomy & & & & & & & & \\
\hline Location & & & & & 0.681 & 0.534 & 0.502 & 0.734 \\
\hline Pancreas & $65(42.2)$ & $10(50.0)$ & $40(43.5)$ & $15(35.7)$ & & & & \\
\hline Common bile duct & $48(31.2)$ & $4(20.0)$ & $28(30.4)$ & $16(38.1)$ & & & & \\
\hline Ampulla of Vater & $38(24.7)$ & $5(25.0)$ & $23(25.0)$ & $10(23.8)$ & & & & \\
\hline Duodenum & 3 (1.9) & $1(5.0)$ & $1(1.1)$ & $1(2.4)$ & & & & \\
\hline Malignancy & & & & & 0.075 & 0.065 & 0.078 & 0.817 \\
\hline Benign & $32(20.8)$ & $8(40.0)$ & $16(17.4)$ & $8(19.0)$ & & & & \\
\hline Malignant & $122(79.2)$ & $12(60.0)$ & 76 (82.6) & $34(81.0)$ & & & & \\
\hline Stage & $122(100)$ & $12(100)$ & $76(100)$ & $34(100)$ & 0.997 & 0.285 & 0.685 & 0.836 \\
\hline I & $36(23.4)$ & 4 (33.3) & $22(28.9)$ & $10(29.4)$ & & & & \\
\hline II & 83 (53.9) & $7(58.3)$ & $53(69.7)$ & $23(67.6)$ & & & & \\
\hline III & $3(1.9)$ & $1(8.3)$ & $1(1.3)$ & $1(2.9)$ & & & & \\
\hline
\end{tabular}

Data are presented as the mean \pm SD or number (\%). Group A, well-nourished; group B, at-risk-of-malnutrition; group C, malnourished. BMI, body mass index; QOL, quality of life.

*Significantly different. 


\section{Statistical analysis}

Data were analyzed using SPSS software version 22.0 (IBM Corp., Armonk, NY, USA). All laboratory values were analyzed as continuous variables. The t-test and analysis of variance tests were performed for normally distributed variables. The MannWhitney U and Kruskal-Wallis tests were performed for nonnormally distributed variables. Categorical data were represented as percentages, and the values were compared using the Pearson chi-square or Fisher exact test. Univariate and multivariate logistic regression analyses were performed to determine the factors affecting overall postoperative morbidity. Variables showing p-values $<0.05$ using univariate analysis were subjected to multivariate analysis using a logistic regression model.

\section{RESULTS}

\section{Patient demographics}

We categorized 154 patients into three groups based on their preoperative nutritional status using the MNA tool as wellnourished ( $n=20,13.0 \%)$, at-risk-of-malnutrition ( $n=92,59.7 \%)$, and malnourished $(n=42,27.3 \%)$. No significant intergroup differences were observed in age; sex; ASA scores; preoperative diabetes mellitus incidence; serum transferrin, serum prealbumin, serum albumin, and serum total protein levels; and cognitive function score (Table 1). However, significant intergroup differences were observed in the preoperative body mass index
(BMI) $\left(25.6 \pm 2.4 \mathrm{~kg} / \mathrm{m}^{2}\right.$ [well-nourished], $23.4 \pm 2.6 \mathrm{~kg} / \mathrm{m}^{2}$ [at-riskof-malnutrition], and $21.1 \pm 2.8 \mathrm{~kg} / \mathrm{m}^{2}$ [malnourished], $\mathrm{p}<0.001$ ). Additionally, significant intergroup differences were observed in the QOL scores $(71.5 \pm 18.5$ [well-nourished], 57.9 \pm 25.9 [at-riskof-malnutrition], and 31.1 \pm 23.2 [malnourished], $\mathrm{p}<0.001$ ). Table 1 shows the perioperative and pathological outcomes based on the preoperative nutritional status of the patients. No significant intergroup differences were observed in the type of surgery, and most patients underwent PPPD ( $\mathrm{n}=135,87.7 \%)$. Similarly, no significant intergroup differences were observed in tumor location, pancreatic texture, intraoperative blood loss, operation time, and the diameter of the main pancreatic duct. The most common location of involvement was the pancreas $(n=65$, 42.2\%), followed by the CBD ( $n=48,31.2 \%)$, AoV ( $n=38,24.7 \%)$, and duodenum ( $\mathrm{n}=3,1.9 \%)$. No significant intergroup difference was observed in terms of malignancy. The percentage of malignant neoplasms was higher than that of benign neoplasms ( $n=122,79.2 \%$ vs $n=32,20.8 \%$ ).

\section{Postoperative outcomes and morbidity}

Table 2 shows the correlation between postoperative morbidity and length of hospitalization and the preoperative nutritional status. A statistically significant difference in overall morbidity was observed between the well-nourished and malnourished groups ( $n=4,20 \%$ vs $n=21,50.0 \%, p=0.026)$. The overall POPF rate was $43.5 \%(n=67)$, and the rate of clinically significant POPF (defined as grade B or C) was 19.5\% $(n=30)$.

Table 2. Postoperative Outcomes According to Preoperative Nutritional Status

\begin{tabular}{|c|c|c|c|c|c|c|c|c|}
\hline & \multirow{2}{*}{$\begin{array}{l}\text { Overall } \\
(n=154)\end{array}$} & \multirow{2}{*}{$\begin{array}{c}\text { Group A } \\
(n=20)\end{array}$} & \multirow{2}{*}{$\begin{array}{c}\text { Group B } \\
(n=92)\end{array}$} & \multirow{2}{*}{$\begin{array}{c}\text { Group C } \\
(\mathrm{n}=42)\end{array}$} & \multicolumn{4}{|c|}{ p-value } \\
\hline & & & & & Total & A vs B & A vs $\mathrm{C}$ & $\mathrm{B}$ vs $\mathrm{C}$ \\
\hline Overall morbidity & $64(41.6)$ & $4(20.0)$ & $39(42.4)$ & $21(50.0)$ & $0.043^{*}$ & 0.062 & $0.026^{*}$ & 0.411 \\
\hline \multicolumn{9}{|l|}{ Clavien-Dindo criteria } \\
\hline Grade II & $25(16.2)$ & 0 & $16(17.4)$ & $9(21.4)$ & $0.041^{*}$ & $0.045^{*}$ & $0.025^{*}$ & 0.578 \\
\hline Grade IIIa or worse & $39(25.3)$ & $4(20.0)$ & $23(25.0)$ & $12(28.6)$ & 0.765 & 0.636 & 0.471 & 0.662 \\
\hline POPF grade & $30(19.5)$ & 0 & $22(23.9)$ & 8 (19.0) & $0.035^{*}$ & $0.015^{*}$ & $0.038^{*}$ & 0.531 \\
\hline Grade B & $28(18.2)$ & 0 & 20 (21.7) & $8(19.0)$ & & & & \\
\hline Grade C & $2(1.3)$ & 0 & $2(2.2)$ & 0 & & & & \\
\hline Delayed gastric emptying & $9(5.8)$ & 0 & $6(6.5)$ & $3(7.1)$ & 0.487 & 0.240 & 0.220 & 0.894 \\
\hline Wound dehiscence & $22(14.3)$ & $4(20.0)$ & $11(12.0)$ & 7 (16.7) & 0.569 & 0.338 & 0.748 & 0.458 \\
\hline Postoperative bleeding & $5(3.2)$ & 0 & $4(4.3)$ & $1(2.4)$ & 0.571 & 0.342 & 0.487 & 0.577 \\
\hline Pulmonary & $3(1.9)$ & 0 & $3(3.3)$ & 0 & 0.359 & 0.412 & & 0.237 \\
\hline Adhesive ileus & $1(0.6)$ & 0 & $1(1.1)$ & 0 & 0.714 & 0.640 & & 0.498 \\
\hline Choledochojejunostomy leak & $1(0.6)$ & 0 & $1(1.1)$ & 0 & 0.714 & 0.640 & & 0.498 \\
\hline Intra-abdominal fluid collection $^{\dagger}$ & $16(10.4)$ & 0 & $10(10.9)$ & $6(14.3)$ & 0.222 & 0.122 & 0.075 & 0.572 \\
\hline In hospital mortality & 0 & 0 & 0 & 0 & & & & \\
\hline Postoperative hospital stay, day & $17.7 \pm 8.3$ & $16.9 \pm 5.2$ & $17.7 \pm 7.9$ & $18.1 \pm 10.2$ & 0.939 & 0.669 & 0.641 & 0.832 \\
\hline
\end{tabular}

Data are presented as the number (\%) or mean \pm SD. Group A, well-nourished; group B, at-risk-of-malnutrition; group C, malnourished. POPF, perioperative pancreatic fistula.

*Significantly different; ${ }^{\dagger}$ Only complications requiring interventional radiologic procedures were counted. 
Significant intergroup differences were observed in the rates of clinically significant POPF (0.0\% [well-nourished] vs 23.9\% [at-risk-of-malnutrition] vs 19.0\% [malnourished], $\mathrm{p}=0.035$ ). Grade C POPF occurred in two patients in the at-risk group; one patient required additional radiological intervention for superior mesenteric artery pseudoaneurysmal bleeding, and one patient required insertion of a percutaneous drain to control POPF followed by admission to the intensive care unit to treat septic shock. However, no inhospital mortality associated with surgical complications was reported.

No significant intergroup differences were observed in terms of other complications. The mean length of hospitalization was the greatest in the malnourished group $(18.1 \pm 10.2$ days $)$, followed by the at-risk-of-malnutrition group (17.7 \pm 7.9 days), and the well-nourished group (16.9 \pm 5.2 days), although this difference was statistically insignificant.

Overall, 38 patients (24.7\%) had a BMI $\geq 25 \mathrm{~kg} / \mathrm{m}^{2}$; among them, 17 patients experienced postoperative morbidities (as against 47 patients with $\mathrm{BMI}<25 \mathrm{~kg} / \mathrm{m}^{2}, \mathrm{p}=0.706$ ). In total, 13 patients experienced postoperative morbidities of grade III or more (as against 26 patients with BMI $<25 \mathrm{~kg} / \mathrm{m}^{2}, \mathrm{p}=0.196$ ).
No significant intergroup differences were observed in terms of postoperative morbidities between patients with BMI $<25 \mathrm{~kg} / \mathrm{m}^{2}$ $(\mathrm{n}=116,75.3 \%)$ and those with BMI $\geq 25 \mathrm{~kg} / \mathrm{m}^{2}$ ( $\left.\mathrm{n}=38,24.7 \%\right)$.

Subgroup analysis was performed on the basis of age categorization to evaluate whether age affected the clinical outcomes (Table 3); 117 patients were aged between 65 and 74 years (76.0\%), and 37 patients were aged between 75 and 85 years (24.0\%). No significant intergroup differences were observed in sex, ASA scores, preoperative diabetes mellitus incidence, and BMI; cognitive function score; QOL scores; nutritional status; and overall morbidity.

\section{Risk factors for overall postoperative morbidity}

Patients were divided into two groups according to the occurrence of postoperative morbidities. Univariate and multivariate analyses of demographic factors, perioperative outcomes, and pathological parameters (Table 4) showed that the at-risk-ofmalnutrition or malnourished status (hazard ratio [HR], 3.45; $\mathrm{p}=0.037)$ and intraoperative blood loss $(\mathrm{HR}, 1.01 ; \mathrm{p}=0.040)$ were significantly associated with overall postoperative morbidity in elderly patients. However, other factors did not significantly

Table 3. Subgroup Analysis According to Age Population

\begin{tabular}{|c|c|c|c|c|}
\hline & \multirow{2}{*}{ Total $(n=154)$} & \multicolumn{2}{|c|}{ Subgrouping according to age, yr } & \multirow{2}{*}{ p-value } \\
\hline & & $65-74(n=117)$ & $75-85(n=37)$ & \\
\hline Sex & & & & 0.708 \\
\hline Male & $80(51.9)$ & $62(53.0)$ & $18(48.6)$ & \\
\hline Female & $74(48.1)$ & $55(47.0)$ & $19(48.1)$ & \\
\hline ASA grade & & & & 0.680 \\
\hline I & $45(29.2)$ & $33(28.2)$ & $12(32.4)$ & \\
\hline II & $109(70.8)$ & $84(71.8)$ & $25(67.6)$ & \\
\hline Preoperative diabetes mellitus & & & & 0.421 \\
\hline No & $108(70.1)$ & $80(68.4)$ & $28(75.7)$ & \\
\hline Yes & $46(29.9)$ & 37 (31.6) & $9(24.3)$ & \\
\hline Preoperative BMI, kg/m² & $23.0 \pm 2.9$ & $23.2 \pm 2.9$ & $22.6 \pm 3.2$ & 0.348 \\
\hline Cognitive score & $86.0 \pm 17.1$ & $85.4 \pm 17.4$ & $88.1 \pm 15.9$ & 0.415 \\
\hline QOL score & $52.5 \pm 27.8$ & $52.9 \pm 28.1$ & $51.1 \pm 27.2$ & 0.734 \\
\hline Nutritional status & & & & 0.191 \\
\hline Well-nourished & $20(13.0)$ & $18(15.4)$ & $2(5.4)$ & \\
\hline At risk & $92(59.7)$ & $70(59.8)$ & $22(59.5)$ & \\
\hline Malnourished & $42(27.3)$ & $29(24.8)$ & $13(35.1)$ & \\
\hline Overall morbidity & & & & 0.703 \\
\hline No & $90(58.4)$ & $67(57.3)$ & $23(62.2)$ & \\
\hline Yes & 64 (41.6) & $50(42.7)$ & $14(37.8)$ & \\
\hline \multicolumn{5}{|l|}{ Clavien-Dindo criteria } \\
\hline Grade II & $25(16.2)$ & $19(16.2)$ & $9(16.2)$ & 0.997 \\
\hline Grade IIIa or worse & 39 (25.3) & $31(26.5)$ & $8(21.6)$ & 0.667 \\
\hline
\end{tabular}

Data are presented as the number (\%) or mean \pm SD.

ASA, American Society of Anesthesiologists; BMI, body mass index; QOL, quality of life. 
Table 4. Univariate and Multivariate Analyses of Risk Factors for Postoperative Overall Morbidity of Elderly Patients with Periampullary Neoplasms

\begin{tabular}{|c|c|c|c|c|c|c|c|}
\hline \multirow[b]{2}{*}{ Variable } & \multirow{2}{*}{$\begin{array}{c}\text { Total } \\
(\mathrm{n}=154)\end{array}$} & \multicolumn{2}{|c|}{ Overall morbidity } & \multicolumn{2}{|c|}{ Univariate analysis } & \multicolumn{2}{|c|}{ Multivariate analysis } \\
\hline & & $\begin{array}{l}\text { Negative } \\
(\mathrm{n}=90)\end{array}$ & $\begin{array}{l}\text { Positive } \\
(\mathrm{n}=64)\end{array}$ & HR $(95 \%$ CI) & p-value & HR $(95 \%$ CI) & $\mathrm{p}$-value \\
\hline Age $\geq 75$ yr & $37(24.0)$ & $23(25.6)$ & $14(21.9)$ & $0.82(0.38-1.74)$ & 0.599 & & \\
\hline Male sex & $80(51.9)$ & $44(48.9)$ & $36(56.3)$ & $1.34(0.71-2.56)$ & 0.368 & & \\
\hline Nutritional status (at risk or malnourished) & $134(87.0)$ & $74(82.2)$ & $60(93.8)$ & $3.24(1.03-10.2)$ & $0.044^{*}$ & $3.45(1.10-11.09)$ & $0.037^{*}$ \\
\hline ASA, grade II & $109(70.8)$ & $66(73.3)$ & $43(67.2)$ & $0.74(0.37-1.50)$ & 0.409 & & \\
\hline Preoperative diabetes mellitus & 46 (29.9) & $26(28.9)$ & $20(31.3)$ & $1.12(0.56-2.25)$ & 0.752 & & \\
\hline Type of surgery, PPPD & $135(87.7)$ & $76(84.4)$ & $59(92.2)$ & $2.46(0.79-7.60)$ & 0.117 & & \\
\hline Malignancy (malignant) & $122(79.2)$ & $68(75.6)$ & $54(84.4)$ & $1.75(0.76-4.00)$ & 0.187 & & \\
\hline Pancreatic texture, soft & $112(72.7)$ & $66(73.3)$ & 46 (71.9) & $1.00(0.45-2.22)$ & 0.986 & & \\
\hline Intraoperative blood loss, $\mathrm{mL}$ & $350 \pm 266$ & $309 \pm 184$ & $407 \pm 343$ & $1.00(1.00-1.03)$ & $0.045^{*}$ & $1.01(1.00-1.03)$ & $0.040^{*}$ \\
\hline Operation time, $\min$ & $305 \pm 90$ & $297 \pm 78$ & $317 \pm 103$ & $1.00(0.99-1.01)$ & 0.998 & & \\
\hline Pancreatic duct diameter, $<3 \mathrm{~mm}$ & $63(40.9)$ & 39 (43.3) & $24(37.5)$ & $1.20(0.58-2.46)$ & 0.613 & & \\
\hline
\end{tabular}

Data are presented as the number (\%) or mean \pm SD.

HR, hazard ratio; CI, confidence interval; ASA, American Society of Anesthesiologists physical status; PPPD, pylorus-preserving pancreatoduodenectomy.

*Significantly different.

Table 5. Change in Quality of Life Score According to Time after Surgery

\begin{tabular}{|c|c|c|c|c|c|c|c|c|}
\hline & \multirow{2}{*}{$\begin{array}{c}\text { Overall } \\
(n=154)\end{array}$} & \multirow{2}{*}{$\begin{array}{c}\text { Group A } \\
(\mathrm{n}=20)\end{array}$} & \multirow{2}{*}{$\begin{array}{c}\text { Group B } \\
(n=92)\end{array}$} & \multirow{2}{*}{$\begin{array}{c}\text { Group C } \\
(n=42)\end{array}$} & \multicolumn{4}{|c|}{$\mathrm{p}$-value } \\
\hline & & & & & Total $^{*}$ & A vs B & A vs C & B vs C \\
\hline \multicolumn{9}{|l|}{ QOL score } \\
\hline Preoperative & $52.5 \pm 27.8$ & $71.5 \pm 18.5$ & $57.9 \pm 25.9$ & $31.1 \pm 23.2$ & $<0.001^{\dagger}$ & $0.032^{\dagger}$ & $<0.001^{\dagger}$ & $<0.001^{\dagger}$ \\
\hline Postoperative 3 mo & $61.3 \pm 19.2$ & $67.6 \pm 16.1$ & $60.8 \pm 20.6$ & $58.8 \pm 16.6$ & 0.220 & 0.148 & 0.083 & 0.605 \\
\hline Postoperative 6 mo & $61.2 \pm 20.3$ & $67.6 \pm 14.9$ & $63.8 \pm 20.6$ & $51.7 \pm 19.8$ & $0.014^{\dagger}$ & 0.392 & $0.004^{\dagger}$ & $0.009^{\dagger}$ \\
\hline Postoperative $12 \mathrm{mo}$ & $67.6 \pm 17.2$ & $71.7 \pm 18.4$ & $67.5 \pm 17.3$ & $65.6 \pm 16.7$ & 0.625 & 0.455 & 0.327 & 0.637 \\
\hline
\end{tabular}

Data are presented as the mean \pm SD. Group A, well-nourished; group B, at-risk-of-malnutrition; group C, malnourished.

*Kruskal-Wallis tests were conducted for variables with nonnormally distributed data; ${ }^{\dagger}$ Significantly different.

differ between patients without and with overall postoperative overall morbidity.

\section{Changes in QOL scores based on postoperative time of assessment}

QOL scores (Table 5) were recorded before and at 3, 6, and 12 months after surgery. The QOL score did not significantly differ before and after surgery in the well-nourished group; however, it increased with time postoperatively in the at-riskof-malnutrition and malnourished groups. The QOL score in the well-nourished and at-risk-of-malnutrition groups was significantly higher than that in the malnourished group 6 months after surgery; however, no significant intergroup differences were observed at 12 months after surgery.

\section{DISCUSSION}

This prospective cohort study categorized elderly patients based on their preoperative nutritional status using the MNA tool to evaluate the effect of preoperative nutritional status on postoperative morbidity in elderly patients with periampullary neoplasms who underwent PD or PPPD. Approximately 87.0\% of the patients had preoperative nutritional deficiencies, and the overall postoperative morbidity rate was higher in patients with nutritional issues than in well-nourished patients. This study indicated that preoperative nutritional issues could affect postoperative outcomes.

The MNA is a validated nutritional screening tool that is particularly useful in elderly patients. ${ }^{10}$ In this study, using the MNA, we observed that 25\% of the patients were malnourished, and the rates of incidence of preoperative malnutrition and atrisk-of-malnutrition were approximately $87 \%$, respectively. A 
previous study also showed similar results, wherein over $80 \%$ of patients with a preoperative diagnosis of periampullary carcinoma were classified as moderately to severely malnourished using nutritional screening tools, and patients with a high nutritional risk showed poor postoperative clinical outcomes. ${ }^{15}$ However, the previous retrospective study primarily focused on postoperative wound complications and did not analyze the known risk factors for morbidities related to pancreatic surgery, including pancreatic texture, pancreatic duct size, operation time, and estimated blood loss. ${ }^{15}$

Although periampullary neoplasm, especially pancreatic cancer, is a fast-growing tumor, it is important to improve the nutritional status of the patients before the surgical procedure. As our patients underwent surgery within 2 to 4 weeks of diagnosis, provision of nutritional support to malnourished patients was possible. Moreover, creating an awareness about the nutritional status of patients at the time of diagnosis is important for the healthcare provider, including surgeon, as well as for the patients and their family.

In this study, 64 patients (41.6\%) experienced postoperative complications and 39 patients (25.3\%) experienced complications classified as $\geq$ grade IIIa in the Clavien-Dindo classification. The overall rate of postoperative complications was higher in the at-risk-of-malnutrition and malnourished groups than in the well-nourished group. No significant intergroup differences in morbidity were observed in terms of complications classified as $\geq$ grade IIIa in the Clavien-Dindo classification. However, four patients in the well-nourished group required wound repair secondary to wound dehiscence. No patient in the well-nourished group showed grade B or C POPF. Notably, four patients in the at-risk-of-malnutrition group and one patient in the malnourished group showed postoperative bleeding, requiring a radiological interventional procedure. Patients in the well-nourished group showed a relatively shorter length of hospitalization, which could be attributed to a lesser severity of postoperative complications in this group than the severity of complications observed in the at-risk-of-malnutrition or malnourished group. ${ }^{16}$ In this study, the rate of severe complications (except wound dehiscence) was higher in the at-risk-of-malnutrition and malnourished groups.

Univariate and multivariate analyses of the risk factors for overall postoperative morbidity showed that the at-risk-ofmalnutrition or malnourished status and intraoperative blood loss were significantly associated with overall postoperative morbidity in elderly patients. Preoperative nutritional status could be a significant predictor of postoperative morbidity in elderly patients with periampullary neoplasms. Thus, physicians should screen such patients carefully to manage their preoperative nutritional status. ${ }^{3,17}$

Malnutrition is associated with impaired QOL. ${ }^{6,7}$ In this study, the overall preoperative QOL scores were lower in the malnourished group than in the at-risk-of-malnutrition and well- nourished groups. The overall preoperative QOL score in the malnourished group was 31.0, which was $<50 \%$ of the reference value. ${ }^{18}$ Notably, malnourished elderly patients are predisposed to cognitive impairment and a low QOL. ${ }^{19,20}$ In this study, QOL scores were recorded before surgery, and at 3, 6, and 12 months after surgery. Interestingly, no significant differences were observed in the QOL scores in the well-nourished group; however, the postoperative QOL score increased with time in the at-riskof-malnutrition and malnourished groups.

This study has some limitations. Variables including socioeconomic status, physical function, and depression (which are known factors affecting nutritional status) were not included in the analysis. Patients were classified into three groups according to their preoperative nutritional status. Unfortunately, the number of patients differed between the groups, and a small-sized group could have affected detailed analysis and, consequently, our results. Nevertheless, this study was one of the largest prospective cohort studies among studies conducted in Northeast Asia countries to evaluate the effects of preoperative malnutrition on postoperative outcomes including postoperative morbidity and the length of hospitalization in elderly patients with periampullary neoplasms.

In conclusion, according to the results of this prospective, single-center cohort study that categorized elderly patients with periampullary neoplasms on the basis of preoperative nutritional status using uniform MNA criteria, 87.0\% patients were classified preoperatively as at-risk-of-malnutrition or malnourished. The overall postoperative morbidity rate was higher in patients with nutritional issues than in well-nourished patients. Therefore, it is important to provide nutritional education and perform intervention to improve preoperative nutritional status in elderly patients with nutritional risk who are scheduled for surgery to treat periampullary neoplasms. Further studies should be conducted to evaluate whether these interventions could prevent malnutrition, thereby reducing overall postoperative morbidity rates.

\section{CONFLICTS OF INTEREST}

No potential conflict of interest relevant to this article was reported.

\section{ACKNOWLEDGEMENTS}

This work was supported by the Collaborative Genome Program for Fostering New Post-Genome Industry of the National Research Foundation funded by the Ministry of Science and ICT (NRF-2017M3C9A5031597).

\section{AUTHOR CONTRIBUTIONS}

Conception and design: E.K., J.Y.J. Administrative support: 
E.K., D.H.L., J.Y.J. Provision of study materials or patients: E.K., J.Y.J. Collection and assembly of data: E.K., D.H.L. Data analysis and interpretation: E.K., D.H.L., J.Y.J. Manuscript writing: E.K., D.H.L., J.Y.J. Final approval of manuscript: all authors.

\section{ORCID}

Eunjung Kim https://orcid.org/0000-0001-6727-1065

Doo-Ho Lee https://orcid.org/0000-0002-9939-207X

Jin-Young Jang https://orcid.org/0000-0003-3312-0503

\section{REFERENCES}

1. Winter JM, Cameron JL, Campbell KA, et al. 1423 pancreaticoduodenectomies for pancreatic cancer: a single-institution experience. J Gastrointest Surg 2006;10:1199-1210.

2. Greenblatt DY, Kelly KJ, Rajamanickam V, et al. Preoperative factors predict perioperative morbidity and mortality after pancreaticoduodenectomy. Ann Surg Oncol 2011;18:2126-2135.

3. Lyu Y, Li T, Cheng Y, Wang B, Chen L, Zhao S. Pancreaticojejunostomy versus pancreaticogastrostomy after pancreaticoduodenectomy: an up-to-date meta-analysis of RCTs applying the ISGPS (2016) criteria. Surg Laparosc Endosc Percutan Tech 2018;28:139-146.

4. Makary MA, Winter JM, Cameron JL, et al. Pancreatico-duodenectomy in the very elderly. J Gastrointest Surg 2006;10:347-356.

5. Li CP. Quality of life patterns and survival among older people. J Nurs Res 2013;21:94-109.

6. Gupta D, Lis CG, Granick J, Grutsch JF, Vashi PG, Lammersfeld CA. Malnutrition was associated with poor quality of life in colorectal cancer: a retrospective analysis. J Clin Epidemiol 2006;59:704-709.

7. Nourissat A, Vasson MP, Merrouche Y, et al. Relationship between nutritional status and quality of life in patients with cancer. Eur J Cancer 2008;44:1238-1242.

8. Norman K, Kirchner H, Lochs H, Pirlich M. Malnutrition affects quality of life in gastroenterology patients. World J Gastroenterol 2006;12:3380-3385.

9. Park HK, Lim BK, Choi SH, Lee HR, Lee DS. Verification of the appropriateness when a shortened version of the Mini Nutritional
Assessment (MNA) is applied for determining the malnutrition state of elderly patients. J Korean Soc Parenter Enter Nutr 2009;2:13-18.

10. Guigoz Y. The Mini Nutritional Assessment (MNA) review of the literature: what does it tell us? J Nutr Health Aging 2006;10:466485.

11. Fitzsimmons D, Johnson CD, George S, et al. Development of a disease specific quality of life (QoL) questionnaire module to supplement the EORTC core cancer QoL questionnaire, the QLQ-C30 in patients with pancreatic cancer. EORTC Study Group on Quality of Life. Eur J Cancer 1999;35:939-941.

12. Clavien PA, Barkun J, de Oliveira ML, et al. The Clavien-Dindo classification of surgical complications: five-year experience. Ann Surg 2009;250:187-196.

13. Bassi C, Dervenis C, Butturini G, et al. Postoperative pancreatic fistula: an international study group (ISGPF) definition. Surgery 2005;138:8-13.

14. Park YC, Kim SW, Jang JY, Ahn YJ, Park YH. Factors influencing delayed gastric emptying after pylorus-preserving pancreatoduodenectomy. J Am Coll Surg 2003;196:859-865.

15. La Torre M, Ziparo V, Nigri G, Cavallini M, Balducci G, Ramacciato G. Malnutrition and pancreatic surgery: prevalence and outcomes. J Surg Oncol 2013;107:702-708.

16. Sikder T, Maimon G, Sourial N, et al. Assessing the effect of preoperative nutrition on upper body function in elderly patients undergoing elective abdominal surgery. JPEN J Parenter Enteral Nutr 2018;42:566-572.

17. Gustafsson UO, Ljungqvist 0. Perioperative nutritional management in digestive tract surgery. Curr Opin Clin Nutr Metab Care 2011;14:504-509.

18. Hinz A, Singer S, Brähler E. European reference values for the quality of life questionnaire EORTC QLQ-C30: results of a German investigation and a summarizing analysis of six European general population normative studies. Acta Oncol 2014;53:958-965.

19. Payette H. Nutrition as a determinant of functional autonomy and quality of life in aging: a research program. Can J Physiol Pharmacol 2005;83:1061-1070.

20. Maki Y, Yamaguchi T, Yamagami T, et al. The impact of subjective memory complaints on quality of life in community-dwelling older adults. Psychogeriatrics 2014;14:175-181. 